\title{
Statistical summability through a lacunary sequence in locally solid Riesz spaces
}

\author{
Syed Abdul Mohiuddine* and Mohammed A Alghamdi
}

\section{"Correspondence:}

mohiuddine@gmail.com

Department of Mathematics,

Faculty of Science, King Abdulaziz

University, P.O. Box 80203, Jeddah,

21589, Saudi Arabia

\begin{abstract}
In this paper, we introduce the concepts of lacunary statistical $\tau$-convergence, lacunary statistically $\tau$-bounded and lacunary statistically $\tau$-Cauchy in the framework of locally solid Riesz spaces. We also define a new type of convergence, that is, $S^{*}(\tau)$-convergence in this setup and prove some interesting results related to these notions.

MSC: $40 \mathrm{~A} 35 ; 40 \mathrm{G} 15 ; 46 \mathrm{~A} 40$

Keywords: density; statistical convergence; lacunary sequence; locally solid Riesz space
\end{abstract}

\section{Introduction and preliminaries}

In 1951 Fast [1] presented the following definition of statistical convergence for sequences of real numbers. We shall denote by $\mathbb{N}$ the set of all natural numbers. Let $K \subseteq \mathbb{N}$ and $K_{n}=\{k \leq n: k \in K\}$. Then the natural density of $K$ is defined by $\delta(K)=\lim _{n} n^{-1}\left|K_{n}\right|$ if the limit exists, where the vertical bars indicate the number of elements in the enclosed set. The sequence $x=\left(x_{k}\right)$ is said to be statistically convergent to $L$ if for every $\varepsilon>0$, the set $K_{\varepsilon}:=\left\{k \in \mathbb{N}:\left|x_{k}-L\right| \geq \varepsilon\right\}$ has natural density zero (cf. Fast [1]), i.e., for each $\varepsilon>0$,

$$
\lim _{n} \frac{1}{n}\left|\left\{j \leq n:\left|x_{j}-L\right| \geq \varepsilon\right\}\right|=0 .
$$

In this case, we write $L=s t$ - $\lim x$. Note that every convergent sequence is statistically convergent, but not conversely. For example, suppose that the sequence $x=\left(x_{n}\right)$ is defined as

$$
x=\left(x_{n}\right)= \begin{cases}\sqrt{n}, & \text { if } n \text { is a square } \\ 0, & \text { otherwise }\end{cases}
$$

It is clear that the sequence $x=\left(x_{n}\right)$ is statistically convergent to 0 , but it is not convergent.

In 1985 Fridy [2] presented the notion of a statistically Cauchy sequence and proved that it is equivalent to statistical convergence. Active research on this topic was started after the papers of Fridy. Mursaleen and Edely [3] extended these concepts from single sequences to double sequences by using two dimensional analogue of natural density. In the recent past, Mursaleen and Mohiuddine [4,5] defined these notions for double sequences in locally solid Riesz spaces as well as in intuitionistic fuzzy normed spaces and proved some 
interesting results. Subsequently, the statistical convergence for sequences of real numbers in several spaces has been extensively investigated by a number of authors, and there are many interesting results concerning this concept. For more details related to this concept, we refer to [6-24] and references therein.

Now, we recall some basic definitions and notions related to the concept of locally solid Riesz spaces. Let $X$ be a real vector space and $\leq$ be a partial order on this space. Then $X$ is said to be an ordered vector space if it satisfies the following properties:

(i) if $x, y \in X$ and $y \leq x$, then $y+z \leq x+z$ for each $z \in X$.

(ii) if $x, y \in X$ and $y \leq x$, then $\lambda y \leq \lambda x$ for each $\lambda \geq 0$.

If in addition $X$ is a lattice with respect to the partial order $\leq$, then $X$ is said to be a Riesz space (or a vector lattice) [25].

For an element $x$ of a Riesz space $X$, the positive part of $x$ is defined by $x^{+}=x \vee \theta=$ $\sup \{x, \theta\}$, the negative part of $x$ by $x^{-}=(-x) \vee \theta$ and the absolute value of $x$ by $|x|=x \vee(-x)$, where $\theta$ is the zero element of $X$.

A subset $S$ of a Riesz space $X$ is said to be solid if $y \in S$ and $|x| \leq|y|$ implies $x \in S$.

A topological vector space $(X, \tau)$ is a vector space $X$, which has a (linear) topology $\tau$, such that the algebraic operations of addition and scalar multiplication in $X$ are continuous. Continuity of addition means that the function $f: X \times X \rightarrow X$ defined by $f(x, y)=x+y$ is continuous on $X \times X$, and continuity of scalar multiplication means that the function $f: \mathbb{C} \times X \rightarrow X$ defined by $f(\lambda, x)=\lambda x$ is continuous on $\mathbb{C} \times X$.

Every linear topology $\tau$ on a vector space $X$ has a base $\mathcal{N}$ for the neighborhoods of $\theta$ satisfying the following properties:

$\left(\mathrm{C}_{1}\right)$ Each $Y \in \mathcal{N}$ is a balanced set, that is, $\lambda x \in Y$ holds for all $x \in Y$ and every $\lambda \in \mathbb{R}$ with $|\lambda| \leq 1$.

$\left(C_{2}\right)$ Each $Y \in \mathcal{N}$ is an absorbing set, that is, for every $x \in X$, there exists $\lambda>0$ such that $\lambda x \in Y$.

$\left(\mathrm{C}_{3}\right)$ For each $Y \in \mathcal{N}$, there exists some $E \in \mathcal{N}$ with $E+E \subseteq Y$.

A linear topology $\tau$ on a Riesz space $X$ is said to be locally solid [26] if $\tau$ has a base at zero consisting of solid sets. A locally solid Riesz space $(X, \tau)$ is a Riesz space equipped with a locally solid topology $\tau$.

The rest of the paper is organized as follows. In Section 2, first we recall the notion of lacunary sequences and define the concepts of lacunary statistically $\tau$-convergent and lacunary statistically $\tau$-bounded and prove some interesting results. Section 3 is devoted to introduce concept of lacunary statistically $\tau$-Cauchy and to proving that a lacunary statistically $\tau$-convergent sequence is lacunary statistically $\tau$-Cauchy. Also, we define $S_{\theta}^{*}(\tau)$ convergent and prove that it is equivalent to lacunary statistically $\tau$-convergent for a first countable space.

\section{Lacunary statistical $\boldsymbol{\tau}$-convergence}

By a lacunary sequence, we mean an increasing integer sequence $\theta=\left(k_{r}\right)$ such that $k_{0}=0$ and $h_{r}:=k_{r}-k_{r-1} \rightarrow \infty$ as $r \rightarrow \infty$. Throughout this paper, the intervals determined by $\theta$ will be denoted by $I_{r}:=\left(k_{r-1}, k_{r}\right]$, and the ratio $k_{r} / k_{r-1}$ will be abbreviated by $q_{r}$.

Let $K \subseteq \mathbb{N}$. The number

$$
\delta_{\theta}(K)=\lim _{r} \frac{1}{h_{r}}\left|\left\{k \in I_{r}: k \in K\right\}\right|
$$


is said to be the $\theta$-density of $K$, provided the limit exists.

In 1993 Fridy and Orhan [27] defined the concept of lacunary statistical convergence as follows.

Let $\theta$ be a lacunary sequence. Then a sequence $x=\left(x_{k}\right)$ is said to be $S_{\theta}$-convergent to the number $L$ if for every $\epsilon>0$, the set $K(\epsilon)$ has $\theta$-density zero, where

$$
K(\epsilon):=\left\{k \in \mathbb{N}:\left|x_{k}-L\right| \geq \epsilon\right\} .
$$

In this case, we write $S_{\theta}-\lim x=L$ or $x_{k} \rightarrow L\left(S_{\theta}\right)$.

Remarks 2.1 It is well known that every convergent sequence is lacunary statistically convergent, but the converse is not true. For example, let the sequence $x=\left(x_{k}\right)$ be defined by

$$
x_{k}= \begin{cases}k ; & \text { for } k_{r}-\left[\sqrt{h_{r}}\right]+1 \leq k \leq k_{r}, r \in \mathbb{N} \\ 0 ; & \text { otherwise }\end{cases}
$$

Then $x$ is lacunary statistically convergent to 0 , but it is not convergent.

We shall assume throughout this paper that the symbol $\mathcal{N}_{\text {sol }}$ will denote any base at zero consisting of solid sets and satisfying the conditions $\left(C_{1}\right),\left(C_{2}\right)$ and $\left(C_{3}\right)$ in a locally solid topology. For our convenience, here and in what follows, we shall write an LSR-space instead of a locally solid Riesz space.

Definition 2.2 Let $(X, \tau)$ be an LSR-space and $\theta$ be a lacunary sequence. Then a sequence $x=\left(x_{j}\right)$ in $X$ is said to be lacunary statistically $\tau$-convergent (or $S_{\theta}(\tau)$-convergent) to the element $\xi \in X$ if for every $\tau$-neighborhood $U$ of zero, $\delta_{\theta}\left(K_{U}\right)=0$, where $K_{U}=\{j \in \mathbb{N}$ : $\left.x_{j}-\xi \notin U\right\}$, i.e.,

$$
\lim _{r \rightarrow \infty} \frac{1}{h_{r}}\left|\left\{j \in I_{r}: x_{j}-\xi \notin U\right\}\right|=0 .
$$

In this case, we write $S_{\theta}(\tau)-\lim x=\xi$ or $x_{j} \stackrel{S_{\theta}(\tau)}{\longrightarrow} \xi$.

Definition 2.3 Let $(X, \tau)$ be an LSR-space and $\theta$ be a lacunary sequence. We say that a sequence $x=\left(x_{j}\right)$ in $X$ is lacunary statistically $\tau$-bounded (or $S_{\theta}(\tau)$-bounded) if for every $\tau$-neighborhood $U$ of zero there exists some $\lambda>0$ such that the set $M_{U}=\left\{j \in \mathbb{N}: \lambda x_{j} \notin U\right\}$ has $\theta$-density zero (shortly, $\delta_{\theta}\left(M_{U}\right)=0$ ), i.e.,

$$
\lim _{r \rightarrow \infty} \frac{1}{h_{r}}\left|\left\{j \in I_{r}: \lambda x_{j} \notin U\right\}\right|=0 .
$$

Theorem 2.4 Let $(X, \tau)$ be a Hausdorff LSR-space and $\theta$ be a lacunary sequence. Suppose that $x=\left(x_{j}\right)$ and $y=\left(y_{k}\right)$ are two sequences in $X$. Then the following hold:

(i) If $S_{\theta}(\tau)-\lim _{j} x_{j}=\xi_{1}$ and $S_{\theta}(\tau)-\lim _{j} x_{j}=\xi_{2}$, then $\xi_{1}=\xi_{2}$.

(ii) If $S_{\theta}(\tau)-\lim _{j} x_{j}=\xi$, then $S_{\theta}(\tau)-\lim _{j} \alpha x_{j}=\alpha \xi, \alpha \in \mathbb{R}$.

(iii) If $S_{\theta}(\tau)-\lim _{j} x_{j}=\xi$ and $S_{\theta}(\tau)-\lim _{j} y_{j}=\eta$, then $S_{\theta}(\tau)-\lim _{j}\left(x_{j}+y_{j}\right)=\xi+\eta$. 
Proof (i) Suppose that $S_{\theta}(\tau)-\lim _{j} x_{j}=\xi_{1}$ and $S_{\theta}(\tau)-\lim _{j} x_{j}=\xi_{2}$. Let $U$ be any $\tau$-neighborhood of zero. Then there exists $Y \in \mathcal{N}_{\text {sol }}$ such that $Y \subseteq U$. Choose any $E \in \mathcal{N}_{\text {sol }}$ such that $E+E \subseteq Y$. We define the following sets:

$$
\begin{aligned}
& K_{1}=\left\{j \in \mathbb{N}: x_{j}-\xi_{1} \in E\right\}, \\
& K_{2}=\left\{j \in \mathbb{N}: x_{j}-\xi_{2} \in E\right\} .
\end{aligned}
$$

Since $S_{\theta}(\tau)-\lim _{j} x_{j}=\xi_{1}$ and $S_{\theta}(\tau)-\lim _{j} x_{j}=\xi_{2}$, we have $\delta_{\theta}\left(K_{1}\right)=\delta_{\theta}\left(K_{2}\right)=1$. Thus $\delta_{\theta}\left(K_{1} \cap\right.$ $\left.K_{2}\right)=1$, and in particular $K_{1} \cap K_{2} \neq \emptyset$. Now, let $j \in K_{1} \cap K_{2}$. Then

$$
\xi_{1}-\xi_{2}=\xi_{1}-x_{j}+x_{j}-\xi_{2} \in E+E \subseteq Y \subseteq U .
$$

Hence, for every $\tau$-neighborhood $U$ of zero, we have $\xi_{1}-\xi_{2} \in U$. Since $(X, \tau)$ is Hausdorff, the intersection of all $\tau$-neighborhoods $U$ of zero is the singleton set $\{\Theta\}$. Thus, we get $\xi_{1}-\xi_{2}=\Theta$, i.e. $\xi_{1}=\xi_{2}$.

(ii) Let $U$ be an arbitrary $\tau$-neighborhood of zero and $S_{\theta}(\tau)-\lim _{j} x_{j}=\xi$. Then there exists $Y \in \mathcal{N}_{\text {sol }}$ such that $Y \subseteq U$ and also

$$
\lim _{r \rightarrow \infty} \frac{1}{h_{r}}\left|\left\{j \in I_{r}: x_{j}-\xi \in Y\right\}\right|=1 .
$$

Since $Y$ is balanced, $x_{j}-\xi \in Y$ implies $\alpha\left(x_{j}-\xi\right) \in Y$ for every $\alpha \in \mathbb{R}$ with $|\alpha| \leq 1$. Hence,

$$
\begin{aligned}
\left\{j \in \mathbb{N}: x_{j}-\xi \in Y\right\} & \subseteq\left\{j \in \mathbb{N}: \alpha x_{j}-\alpha \xi \in Y\right\} \\
& \subseteq\left\{j \in \mathbb{N}: \alpha x_{j}-\alpha \xi \in U\right\} .
\end{aligned}
$$

Thus, we obtain

$$
\lim _{r \rightarrow \infty} \frac{1}{h_{r}}\left|\left\{j \in I_{r}: \alpha x_{j}-\alpha \xi \in U\right\}\right|=1,
$$

for each $\tau$-neighborhood $U$ of zero. Now, let $|\alpha|>1$ and $[|\alpha|]$ be the smallest integer greater than or equal to $|\alpha|$. There exists $E \in \mathcal{N}_{\text {sol }}$ such that $[|\alpha|] E \subseteq Y$. Since $S_{\theta}(\tau)-\lim _{j} x_{j}=\xi$, the set

$$
K=\left\{j \in \mathbb{N}: x_{j}-\xi \in E\right\}
$$

has $\theta$-density zero. Therefore,

$$
\left|\alpha \xi-\alpha x_{j}\right|=|\alpha|\left|\xi-x_{j}\right| \leq[|\alpha|]\left|\xi-x_{j}\right| \in[|\alpha|] E \subseteq Y \subseteq U
$$

Since the set $Y$ is solid, we have $\alpha \xi-\alpha x_{j} \in Y$. This implies that $\alpha \xi-\alpha x_{j} \in U$. Thus,

$$
\lim _{r \rightarrow \infty} \frac{1}{h_{r}}\left|\left\{j \in I_{r}: \alpha x_{j}-\alpha \xi \in U\right\}\right|=1,
$$

for each $\tau$-neighborhood $U$ of zero. Hence, $S_{\theta}(\tau)-\lim _{j} \alpha x_{j}=\alpha \xi$. 
(iii) Let $U$ be an arbitrary $\tau$-neighborhood of zero. Then there exists $Y \in \mathcal{N}_{\text {sol }}$ such that $Y \subseteq U$. Choose $E$ in $\mathcal{N}_{\text {sol }}$ such that $E+E \subseteq Y$. Since $S_{\theta}(\tau)-\lim _{j} x_{j}=\xi$ and $S_{\theta}(\tau)-\lim _{j, k} y_{j k}=$ $\eta$, we have $\delta_{\theta}\left(H_{1}\right)=1=\delta_{\theta}\left(H_{2}\right)$, where

$$
\begin{aligned}
& H_{1}=\left\{j \in \mathbb{N}: x_{j}-\xi \in E\right\}, \\
& H_{2}=\left\{j \in \mathbb{N}: y_{j}-\eta \in E\right\} .
\end{aligned}
$$

Let $H=H_{1} \cap H_{2}$. Hence, we have $\delta_{\theta}(H)=1$ and

$$
\left(x_{j}+y_{j}\right)-(\xi+y)=\left(x_{j}-x\right)+\left(y_{j}-\eta\right) \in E+E \subseteq Y \subseteq U .
$$

Therefore,

$$
\lim _{r \rightarrow \infty} \frac{1}{h_{r}}\left|\left\{j \in I_{r}:\left(x_{j}+y_{j}\right)-(\xi+\eta) \in U\right\}\right|=1 .
$$

Since $U$ is arbitrary, we have $S_{\theta}(\tau)-\lim _{j}\left(x_{j}+y_{j}\right)=\xi+\eta$.

Theorem 2.5 Let $(X, \tau)$ be an LSR-space and $\theta$ be a lacunary sequence. If a sequence $x=$ $\left(x_{j}\right)$ is lacunary statistically $\tau$-convergent, then it is lacunary statistically $\tau$-bounded.

Proof Suppose $x=\left(x_{j}\right)$ is lacunary statistically $\tau$-convergent to the point $\xi \in X$ and let $U$ be an arbitrary $\tau$-neighborhood of zero. Then there exists $Y \in \mathcal{N}_{\text {sol }}$ such that $Y \subseteq U$. Let us choose $E \in \mathcal{N}_{\text {sol }}$ such that $E+E \subseteq Y$. Since $S_{\theta}(\tau)-\lim _{j \rightarrow \infty} x_{j}=\xi$, the set

$$
K=\left\{j \in \mathbb{N}: x_{j}-\xi \notin E\right\}
$$

has $\theta$-density zero. Since $E$ is absorbing, there exists $\lambda>0$ such that $\lambda \xi \in E$. Let $\alpha$ be such that $\alpha \leq 1$ and $\alpha \leq \lambda$. Since $E$ is solid and $|\alpha \xi| \leq|\lambda \xi|$, we have $\alpha \xi \in E$. Since $E$ is balanced, $x_{j}-\xi \in E$ implies $\alpha\left(x_{j}-\xi\right) \in E$. Then we have

$$
\alpha x_{j}=\alpha\left(x_{j}-\xi\right)+\alpha \xi \in E+E \subseteq Y \subseteq U,
$$

for each $j \in \mathbb{N} \backslash K$. Thus,

$$
\lim _{r \rightarrow \infty} \frac{1}{h_{r}}\left|\left\{j \in I_{r}: \alpha x_{j} \notin U\right\}\right|=0 .
$$

Hence, $\left(x_{j}\right)$ is lacunary statistically $\tau$-bounded.

Theorem 2.6 Let $(X, \tau)$ be an LSR-space and $\theta$ be a lacunary sequence. If $\left(x_{j}\right),\left(y_{j}\right)$ and $\left(z_{j}\right)$ are three sequences such that

(i) $x_{j} \leq y_{j} \leq z_{j}$, for all $j \in \mathbb{N}$,

(ii) $S_{\theta}(\tau)-\lim _{j} x_{j}=\xi=S_{\theta}(\tau)-\lim _{j} z_{j}$, then $S_{\theta}(\tau)-\lim _{j} y_{j}=\xi$. 
Proof Let $U$ be an arbitrary $\tau$-neighborhood of zero, there exists $Y \in \mathcal{N}_{\text {sol }}$ such that $Y \subseteq U$. Choose $E \in \mathcal{N}_{\text {sol }}$ such that $E+E \subseteq Y$. From the condition (ii), we have $\delta_{\theta}(A)=$ $1=\delta_{\theta}(B)$, where

$$
\begin{aligned}
& A=\left\{j \in \mathbb{N}: x_{j}-\xi \in E\right\}, \\
& B=\left\{j \in \mathbb{N}: z_{j}-\xi \in E\right\} .
\end{aligned}
$$

Also, we get $\delta_{\theta}(A \cap B)=1$, and from (i), we have

$$
x_{j}-\xi \leq y_{j}-\xi \leq z_{j}-\xi
$$

for all $j \in \mathbb{N}$. This implies that for all $j \in A \cap B$, we get

$$
\left|y_{j}-\xi\right| \leq\left|x_{j}-\xi\right|+\left|z_{j}-\xi\right| \in E+E \subseteq Y .
$$

Since $Y$ is solid, we have $y_{j}-\xi \in Y \subseteq U$. Thus,

$$
\lim _{r \rightarrow \infty} \frac{1}{h_{r}}\left|\left\{j \in I_{r}: y_{j}-\xi \in U\right\}\right|=1,
$$

for each $\tau$-neighborhood $U$ of zero. Hence, $S_{\theta}(\tau)-\lim _{j} y_{j}=\xi$.

\section{Lacunary statistically $\tau$-Cauchy and $S_{\theta}^{*}(\tau)$-convergence}

In the present section, first we define the concept of lacunary statistically $\tau$-Cauchy in locally solid Riesz spaces as follows.

Definition 3.1 Let $(X, \tau)$ be an LSR-space and $\theta$ be a lacunary sequence. A sequence $x=$ $\left(x_{j}\right)$ in $X$ is lacunary statistically $\tau$-Cauchy if for every $\tau$-neighborhood $U$ of zero there exists $p \in \mathbb{N}$ such that

$$
\lim _{r \rightarrow \infty} \frac{1}{h_{r}}\left|\left\{j \in I_{r}: x_{j}-x_{p} \notin U\right\}\right|=0 .
$$

Theorem 3.2 Let $(X, \tau)$ be an LSR-space and $\theta$ be a lacunary sequence. If a sequence $x=$ $\left(x_{j}\right)$ is lacunary statistically $\tau$-convergent, then it is lacunary statistically $\tau$-Cauchy.

Proof Suppose that $S_{\theta}(\tau)-\lim _{j \rightarrow \infty} x_{j}=\xi$. Let $U$ be an arbitrary $\tau$-neighborhood of zero, there exists $Y \in \mathcal{N}_{\text {sol }}$ such that $Y \subseteq U$. Choose $E \in \mathcal{N}_{\text {sol }}$ such that $E+E \subseteq Y$. Then

$$
\lim _{r \rightarrow \infty} \frac{1}{h_{r}}\left|\left\{j \in I_{r}: x_{j}-\xi \notin E\right\}\right|=0 .
$$

Also, we have

$$
x_{j}-x_{p}=x_{j}-\xi+\xi-x_{p} \in E+E \subseteq Y \subseteq U,
$$

for all $j, p \in \mathbb{N} \backslash K$, where

$$
K=\left\{j \in \mathbb{N}: x_{j}-\xi \notin E\right\} .
$$


Therefore, the set

$$
\left\{j \in \mathbb{N}: x_{j}-x_{p} \notin U\right\} \subseteq K
$$

For every $\tau$-neighborhood $U$ of zero, there exists $N \in \mathbb{N}$ such that for all $j, p \geq N$,

$$
\lim _{r} \frac{1}{h_{r}}\left\{j \in I_{r}: x_{j}-x_{p} \notin U\right\}=0 .
$$

Hence, $\left(x_{j}\right)$ is lacunary statistically $\tau$-Cauchy.

Now, we define another type of convergence in locally solid Riesz spaces.

Definition 3.3 Let $\theta$ be a lacunary sequence. A sequence $\left(x_{j}\right)$ in an LSR-space $(X, \tau)$ is said to be $S_{\theta}^{*}(\tau)$-convergent to $\xi \in X$ if there exists an index set $K=\left\{j_{n}\right\} \subseteq \mathbb{N}, n=1,2, \ldots$, with $\delta_{\theta}(K)=1$ such that $\lim _{n \rightarrow \infty} x_{j_{n}}=\xi$. In this case, we write $\xi=S_{\theta}^{*}(\tau)-\lim x$.

Theorem 3.4 Let $\theta$ be a lacunary sequence. A sequence $x=\left(x_{j}\right)$ is lacunary statistically $\tau$-convergent to a number $\xi$ if it is $S_{\theta}^{*}(\tau)$-convergent to $\xi$ in a locally solid Riesz space $(X, \tau)$.

Proof Let $U$ be an arbitrary $\tau$-neighborhood of $\xi$. Since $x=\left(x_{j}\right)$ is $S_{\theta}^{*}(\tau)$-convergent to $\xi$, there is an index set $K=\left\{j_{n}\right\} \subseteq \mathbb{N}, n=1,2, \ldots$, with $\delta_{\theta}(K)=1$ and $j_{0}=j_{0}(U)$, such that $j \geq j_{0}$ and $j \in K$ imply $x_{j}-\xi \in U$. Then

$$
K_{U}=\left\{j \in \mathbb{N}: x_{j}-\xi \notin U\right\} \subseteq \mathbb{N}-\left\{j_{N+1}, j_{N+2}, \ldots\right\}
$$

Therefore,

$$
\delta_{\theta}\left(K_{U}\right) \leq 1-1=0 .
$$

Hence, $x$ is lacunary statistically $\tau$-convergent to $\xi$.

Note that the converse holds for a first countable space.

Recall that a first countable space is a topological space satisfying the 'first axiom of countability'. Specifically, a space $X$ is said to be first countable if each point has a countable neighborhood basis (local base). That is, for each point $x$ in $X$ there exists a sequence $U_{1}, U_{2}, \ldots$ of open neighborhoods of $x$ such that for any open neighborhood $V$ of $x$ there exists an integer $i$ with $U_{i}$ contained in $V$.

Theorem 3.5 Let $(X, \tau)$ be a first countable LSR-space and $\theta$ be a lacunary sequence. If a sequence $x=\left(x_{j}\right)$ is lacunary statistically $\tau$-convergent to a number $\xi$, then it is $S_{\theta}^{*}(\tau)$ convergent to $\xi$.

Proof Let $x$ be lacunary statistically $\tau$-convergent to a number $\xi$. Fix a countable local base $U_{1} \supset U_{2} \supset U_{3} \supset \cdots$ at $\xi$. For every $i \in \mathbb{N}$, put

$$
K_{i}=\left\{j \in \mathbb{N}: x_{j}-\xi \notin U_{i}\right\}
$$


and

$$
M_{i}=\left\{j \in \mathbb{N}: x_{j}-\xi \in U_{i}\right\} \quad(i=1,2,3, \ldots) .
$$

Then $\delta_{\theta}\left(K_{i}\right)=0$ and

(1) $M_{1} \supset M_{2} \supset \cdots \supset M_{i} \supset M_{i+1} \supset \cdots$

and

(2) $\delta_{\lambda}\left(M_{i}\right)=1, i=1,2,3, \ldots$

Now, we have to show that for $j \in M_{i},\left(x_{j_{n}}\right)$ is convergent to $\xi$. Suppose that $\left(x_{j_{n}}\right)$ is not convergent to $\xi$. Therefore, $x_{j_{n}}-\xi \notin U_{i}$ for infinitely many terms. Let

$$
M_{r}=\left\{j \in \mathbb{N}: x_{j_{n}}-\xi \in U_{r}\right\} \quad(r>i) .
$$

Then

(3) $\delta_{\theta}\left(M_{r}\right)=0$,

and by (1), $M_{i} \subset M_{r}$. Hence, $\delta_{\theta}\left(M_{i}\right)=0$, which contradicts (2). Therefore, $\left(x_{j_{n}}\right)$ is convergent to $\xi$. Hence, by Definition 3.3, $x$ is $S_{\theta}^{*}(\tau)$-convergent to $\xi$.

\section{Competing interests}

The authors declare that they have no competing interests.

\section{Authors' contributions}

The authors contributed equally and significantly in writing this paper. Both the authors read and approved the final manuscript.

\section{Acknowledgements}

The authors would like to thank the anonymous reviewers for their valuable comments.

Received: 19 May 2012 Accepted: 21 September 2012 Published: 9 October 2012

\section{References}

1. Fast, H: Sur la convergence statistique. Colloq. Math. 2, 241-244 (1951)

2. Fridy, JA: On statistical convergence. Analysis 5, 301-313 (1985)

3. Mursaleen, M, Edely, OHH: Statistical convergence of double sequences. J. Math. Anal. Appl. 288, $223-231$ (2003)

4. Mohiuddine, SA, Alotaibi, A, Mursaleen, M: Statistical convergence of double sequences in locally solid Riesz spaces Abstr. Appl. Anal. 2012, Article ID 719729 (2012)

5. Mursaleen, M, Mohiuddine, SA: Statistical convergence of double sequences in intuitionistic fuzzy normed spaces. Chaos Solitons Fractals 41, 2414-2421 (2009)

6. Albayrak, H, Pehlivan, S: Statistical convergence and statistical continuity on locally solid Riesz spaces. Topol. Appl. 159, 1887-1893 (2012)

7. Cakalli, H: Lacunary statistical convergence in topological groups. Indian J. Pure Appl. Math. 26(2), 113-119 (1995)

8. Çakalli, H: On Statistical Convergence in topological groups. Pure Appl. Math. Sci. 43, 27-31 (1996)

9. Çakalli, H, Khan, MK: Summability in topological spaces. Appl. Math. Lett. 24, 348-352 (2011)

10. Çakalli, H, Savaş, E: Statistical convergence of double sequence in topological groups. J. Comput. Anal. Appl. 12(2), 421-426 (2010)

11. Maddox, IJ: Statistical convergence in a locally convex space. Math. Proc. Camb. Philos. Soc. 104, 141-145 (1988)

12. Di Maio, G, Kočinac, LDR: Statistical convergence in topology. Topol. Appl. 156, 28-45 (2008)

13. Mohiuddine, SA, Aiyub, M: Lacunary statistical convergence in random 2-normed spaces. Appl. Math. Inf. Sci. 6(3), 581-585 (2012)

14. Mohuiddine, SA, Alotaibi, A, Alsulami, SM: Ideal convergence of double sequences in random 2-normed spaces. Adv. Differ. Equ. 2012, 149 (2012)

15. Mohiuddine, SA, Danish Lohani, QM: On generalized statistical convergence in intuitionistic fuzzy normed space. Chaos Solitons Fractals 42, 1731-1737 (2009)

16. Mohiuddine, SA, Şavas, E: Lacunary statistically convergent double sequences in probabilistic normed spaces. Ann. Univ. Ferrara (2012). doi:10.1007/s11565-012-0157-5

17. Mohiuddine, SA, Şevli, H, Cancan, M: Statistical convergence in fuzzy 2-normed space. J. Comput. Anal. Appl. 12(4), 787-798 (2010)

18. Mursaleen, M, Çakan, C, Mohiuddine, SA, Savaş, E: Generalized statistical convergence and statistical core of double sequences. Acta Math. Sin. Engl. Ser. 26, 2131-2144 (2010)

19. Mursaleen, M, Edely, OHH: Generalized statistical convergence. Inf. Sci. 162, 287-294 (2004) 
20. Mursaleen, M, Mohiuddine, SA: On lacunary statistical convergence with respect to the intuitionistic fuzzy normed space. J. Comput. Appl. Math. 233, 142-149 (2009)

21. Mursaleen, M, Mohiuddine, SA: On ideal convergence of double sequences in probabilistic normed spaces. Math. Rep. (Bucur.) 12(64)(4), 359-371 (2010)

22. Mursaleen, M, Mohiuddine, SA: On ideal convergence in probabilistic normed spaces. Math. Slovaca 62, 49-62 (2012)

23. Mursaleen, $\mathrm{M}$, Mohiuddine, SA, Edely, OHH: On the ideal convergence of double sequences in intuitionistic fuzzy normed spaces. Comput. Math. Appl. 59, 603-611 (2010)

24. Savaş, E, Mohiuddine, SA: $\bar{\lambda}$-statistically convergent double sequences in probabilistic normed spaces. Math. Slovaca 62(1), 99-108 (2012)

25. Zaanen, AC: Introduction to Operator Theory in Riesz Spaces. Springer, Berlin (1997)

26. Roberts, GT: Topologies in vector lattices. Math. Proc. Camb. Philos. Soc. 48, 533-546 (1952)

27. Fridy, JA, Orhan, C: Lacunary statistical convergence. Pac. J. Math. 160, 43-51 (1993)

doi:10.1186/1029-242X-2012-225

Cite this article as: Mohiuddine and Alghamdi: Statistical summability through a lacunary sequence in locally solid Riesz spaces. Journal of Inequalities and Applications 2012 2012:225.

\section{Submit your manuscript to a SpringerOpen ${ }^{\ominus}$ journal and benefit from:}

- Convenient online submission

Rigorous peer review

- Immediate publication on acceptance

- Open access: articles freely available online

- High visibility within the field

- Retaining the copyright to your article 